\title{
Efficient Expansion of Human Umbilical Cord Blood- Derived NK Cells Ex Vivo without Requiring Feeder Layers
}

\author{
Chu Thi Thao" ${ }^{1 \#, ~ D o ~ T h i ~ H o a i ~ T h u ~}{ }^{1,2 \#, ~ B u i ~ V i e t ~ A n h}{ }^{1}$, Truong Linh Huyen ${ }^{1}$, Nguyen Van Phong ${ }^{1}$, Nguyen Thanh Liem ${ }^{1}$ \\ and Hoang Thi My Nhung*1,2 \\ ${ }^{1}$ Vinmec Research Institute of Stem cells and Gene Technology, Vietnam \\ ${ }^{2}$ VNU University of Science, Vietnam \\ ${ }^{\#}$ These two authors contributes equally to this manuscript.
}

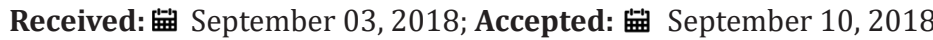

*Corresponding author: Hoang Thi My Nhung, Vinmec Research Institute of Stem cells and Gene Technology, VNU University of Science, Hanoi, Vietnam

\begin{abstract}
Introduction: Natural killer (NK) cells are central components of the innate immunity. They have ability to kill a wide range of cancer cells and are a promising tool for both autologous and allogeneic immune enhancement therapy in cancer treatment. Actually, NK cells can be derived from multiple sources such as: peripheral blood, cord blood... Among these, cord blood (CB) is known as an ideal for NK cells expansion because it occupies a higher percentage of NK cells compare to the peripheral blood and is a rich source of hematopoietic stem cell as well as progenitor immune cells. The objectives of this study were to invent a reasonable approach for expanding a relevant number of NK cells from human cord blood to clinical application. Methods: At the initial step, cord blood was collected from the patients and then the mononuclear cells were obtained by density centrifugation with Ficoll.
\end{abstract}

After that, these cells were cultured in 2 stages: stimulation and expansion with inactivated autologous plasma and BINKIT expansion kit contains several kinds of growth factors which are specified for NK cells. The immunophenotype of NK cells was analyzed every 2 days through \%CD56+CD3- by Flow cytometry technique. The ex-vivo activation and expansion of NK cells was performed in GMP grade -clean room for about 3 weeks. Results: After culturing periods, the average NK cell count post-expansion was $2040.6 \pm 1463.5 \times 10^{\wedge} 6$ and the average cell fold expansion was $814.4 \pm 560.9$, the expanded cells presented $90.6 \% \pm 8.9 \%$ purify of CD56+CD3-, the percentage of CD56 bright CD3- cells was $84.2 \% \pm 14.4 \%$. Conclusion: Human cord blood has a significant potential to expand NK cells to relevant number for clinical use and our method are suitable for getting a relevant number and quality of NK cells to the clinical use.

Keywords: Natural Killer Cells; Cord Blood; Autologous Plasma; Growth Factors; Feeder Layer; Cytotoxic T Cells; Tumour; NK Cocktail; Ficoll Solution; Immunoglobulin

Abbreviations: NK: Natural Killer; UCB: Umbilical Cord Blood; CTL: Cytotoxic T Cells; IFN- $\gamma$ : Interferon Gamma; IL: Interleukin; ILCs: Innate Lymphoid Cells; KIRs: Killer Cell Immunoglobulin (Ig) like Receptors; MHC: Major Histocompatibility Complex; MNC: Mononuclear Cell; PBMCs: Peripheral Blood Mononuclear Cells

\section{Introduction}

Nowadays, cancer is still a leading cause of death in the world. However, the standard treatments for cancer such as surgical therapy, radiotherapy and chemotherapy usually bring multiple side-effects to patients. In some recent years, the invention of cell therapy has opened brighter future in some life-threatening diseases treatment, especially cancer. This approach helps patients to avoid immunodeficiency through sufficient provision of immune cells as well as stimulating these cells to kill specifically cancer cells. Clinical trials of cell therapy for treating many different cancers are currently ongoing. In fact, there are many types of immune cells which are used for cell therapy such as: NK cells, cytotoxic T cells (CTL), dendritic cells (DC), $\gamma \delta$ T cells and $\alpha \beta$ T cells [1]. Natural Killer (NK) cells are a subtype of type 1 ILCs (innate lymphoid cells), which play significant roles in innate immune responses.
NK cells can produce cytokine and have cytotoxicity to kill both viral infected and tumour cells. Because of the ability to secrete immunostimulatory cytokines like IFN- $\gamma$, NK cells can manipulate not only tumour growth but also metastasis [2]. Therefore, NK cells have been known as promising tools for cell therapies in cancer treatment. Initial clinical trials have demonstrated that the infusion of NK cells is entirely possible and safe with no side effects and minimal toxicity to patients [3]. NK cells can be determined by the expression of CD56 and the absence of the T cell marker CD3 on their surface. Besides, most human blood NK cells also express CD16, which is involved in recognition of antibody-coated cells [4]. NK cells can be divided into two main types: CD56bright and CD56dim populations [5,6]. The CD56bright cells have low or absent expression of CD16 and killer-cell immunoglobulin-like 
receptors (KIR), whereas the CD56dim cells express both CD16 and KIR $[7,6]$.

The function of NK cells is regulated through the interaction between activating receptors and inhibitory receptors [4]. The lack of combination between inhibitory KIR receptor on NK cells surface and MHC-I on target cell surface lead to the stimulation of activating receptors, and then helps NK cells to kill target cells. Therefore, the KIR mismatch assists NK cells in expressing more killing activity [8]. This is also the reason why adoptive cell transfer (ACT) approaches using allogeneic NK cells have been more effective for cancer immunotherapy [9]. In order to conduct the clinical application about NK cell-based immunotherapy, it is needs to obtain a sufficient number of NK cells with high cytotoxicity. The sources of NK cells include umbilical cord blood (UCB), bone marrow, peripheral blood (PB) and embryonic stem cells. Actually, UCB not only has higher proportion of NK cells but is also an easy collecting source.

Furthermore, the establishment of UCB bank in the world now assists UCB being preserved for a long time. This leads to UCB can be used as a ready source for NK cell-based therapy. Nevertheless, although UCB contains greater frequencies of NK cells as opposed to $\mathrm{PB}$, the numbers of obtained $\mathrm{NK}$ cells are still really small because of the limited UCB volume. This is a major hindrance in providing adequate numbers of NK cells for clinical trial [10]. The main objectives of this study were to optimize the procedure of expansion of NK cells from human cord blood in order to obtain a larger number of NK cells and higher purify of CD56+CD3- in population. The next stage is activation of these NK cells to increase the capacity of killing cancer cells effectively.

\section{Materials and Methods}

\section{Collection of Cord Blood Samples}

Human cord blood which is collected directly from the umbilical cord of the new born baby at Vinmec International Hospital. Before collection, the mother was diagnosed healthy and does not carry any of the following viruses: HIV, HBV, HPV, HCV. All the pregnant woman singed the written consent which is approved by the Ethics Committee of Vinmec International Hospital.

\section{Isolation and Inactivation of Autologous Plasma}

The whole cord blood was firstly centrifuged at $1700 \times \mathrm{g}$ for 10 minutes. After centrifugation, whole blood is separated into 2 layers: plasma layer above and blood cell layer below. Then, the plasma layer was collected by pipetting. This plasma was then heat inactivated at $58^{\circ} \mathrm{C}$ for 60 minutes. After inactivating, plasma continued to be centrifuged at $1700 \times$ g for 5 minutes to collect the supernatant plasma and discard the pellet at the bottom of the tube.

\section{Isolating Mononuclear Cells}

Cord blood after plasma collection is diluted with PBS, followed by dropping down slightly to Ficoll-Paque/Lymphoprep (Stem Cell
Technologies, CA) with volume proportion 2:1, and then the mixture was density centrifuged at $840 \times \mathrm{g}$ for 20 minutes. The Ficoll solution plays a role as a tool to separate the whole blood into many layers follow the density gradient. After centrifugation, the mixture was divided into 4 layers, from top to bottom: plasma, a layer of mononuclear cells (MNC) called buffy coat, Ficoll, and erythrocytes. The MNC were collected and then washed with PBS 2 times.

\section{Stimulation Culture}

The stimulation culture process was implemented within 3 days (day 0 to day 3). UCB-MNCs were cultured in initial NK medium and initial NK cocktail (Biotherapy Institute Japan) containing IL2, OK432, zoledronic acid and 10\% heat-inactivated autologous plasma in an initial NK flask (Biotherapy Institute Japan) for initial activation. This flask was immobilized with anti-CD3 monoclonal antibody and anti-CD16 monoclonal antibody. The cell density at seeding was $1 \times 106$ cells $/ \mathrm{ml}$.

\section{Expansion Culture}

After 3 days of stimulating cultivation, the cells need to be cultured in new condition which is free from the anti-CD3 antibody, anti-CD16 antibody, OK432, and zoledronic acid or the like. These factors were removed by centrifuging the medium contains cells which has undergone the stimulation step at $270 \mathrm{xg}$ for 8 minutes and removing the supernatant. Then, the cells were transferred to an anti-CD3 antibody and anti-CD16 antibody-uncoated flask and cultured in subculture medium (Biotherapy Institute Japan) supplemented with IL-2 and 10\% heat-inactivated human plasma. Subculture medium was added depending to the cell number every 2 days. The cells were incubated at $37{ }^{\circ} \mathrm{C}$ with $5 \% \mathrm{CO}_{2}$.

\section{Immunophenotyping Analysis by Flowcytometry}

Cells were stained with these following antibodies: Anti-CD3Pacific Blue, Anti-CD4- APC-Alexa Flour 750, Anti-CD8- FITC, AntiCD56-PE, Anti IgG1- Pacific Blue, Anti IgG- APC- Alexa Flour 750, Anti IgG1- FITC, Anti IgG1- PE (Miltenyi Biotec, Germany). Stained cells were analysed using Navios flow cytometer with Navios software (Beckman Coulter, CA).

\section{Data Analysis}

Data was statistically analysed by Microsoft Excel version 2013.

\section{Results}

\section{The Expansion of NK Cell}

The cells at day 0 distributed uniformly in population (Figure 1A). From day 3, there was the appearance of cell clumps (Figure 1B). Cell population reached the log phase from D11 of culture (Fig. 1C). NK cells were not only dominant during cell culture period but also significantly increased the number. The average final total cell counts we obtained from three samples was $2185.3 \times 10^{\wedge} 6$ (ranging from $1102-1651.9 \times 10^{\wedge} 6$ ) and the average cell fold expansion was $42.3 \pm 18.3$. Notably, the average NK cell counts post-expansion we 
obtained was $2040.6 \times 10^{\wedge} 6$ (ranging from $871.7-3681.9 \times 10^{\wedge} 6$ ) The viability of cells at the day of ending culture was $98.6 \% \pm 0.6 \%$. and the average cell fold expansion was $814.4 \pm 560.9$ (Table 1$)$.

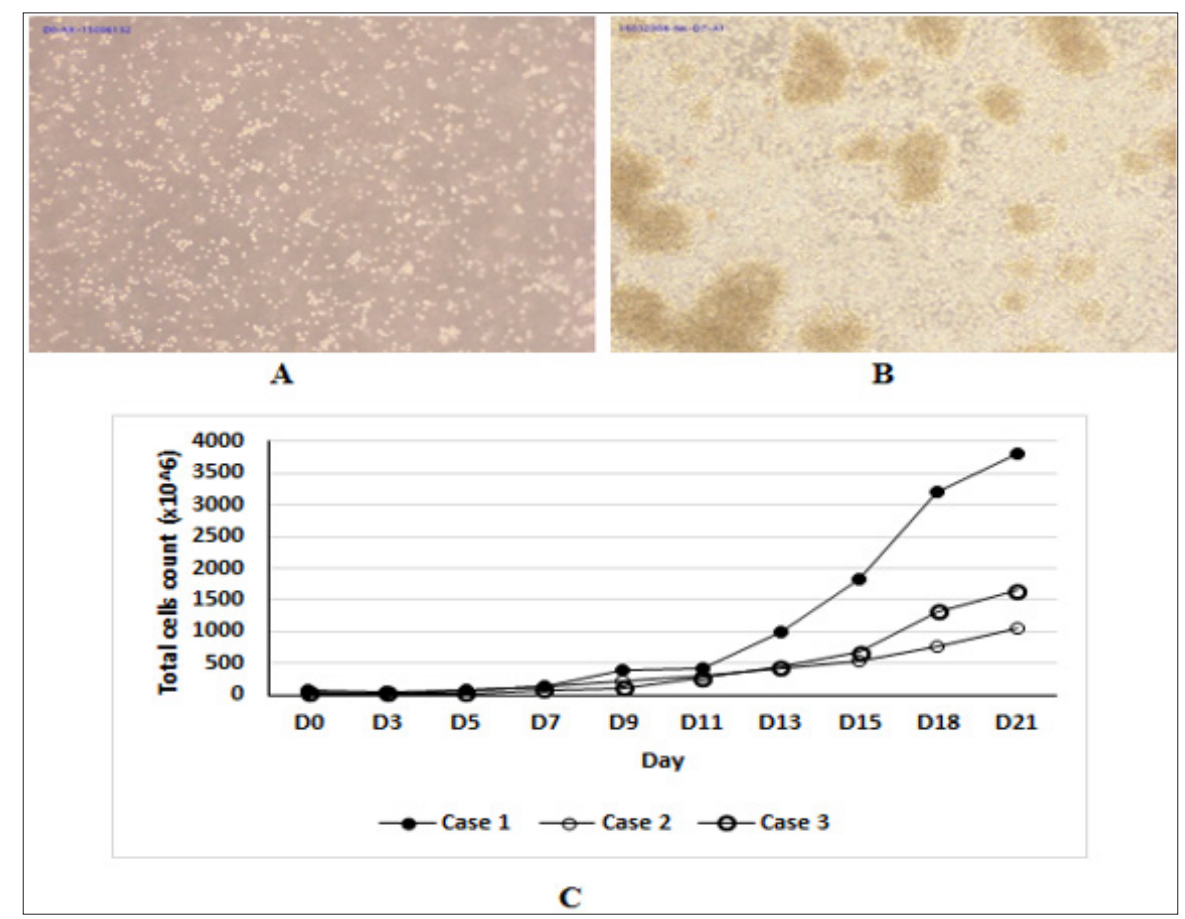

Figure 1: The morphologies of the NK cells and the number of total cells in case 1. (A) The NK cell population at day 0 (Objective 10x). (B) The NK cell population at day 7. There were clumps in cell population (Black arrows) (Objective 10x). (C) The time course of growth cells from three samples for 21 days.

Table 1: The number of cell count pre- and post-expansion.

\begin{tabular}{|c|c|c|c|c|c|c|}
\hline \multirow{2}{*}{ Case } & \multicolumn{2}{|c|}{$\begin{array}{l}\text { Initial cell count } \\
\text { (pre-expansion) }\end{array}$} & \multicolumn{2}{|c|}{$\begin{array}{l}\text { Final cell count } \\
\text { (post-expansion) }\end{array}$} & \multicolumn{2}{|c|}{ Cell fold expansion } \\
\hline & $\begin{array}{l}\text { Total cells } \\
\left(\times 10^{\wedge} 6\right)\end{array}$ & $\begin{array}{l}\text { NK cells } \\
\left(\times 10^{\wedge} 6\right)\end{array}$ & $\begin{array}{l}\text { Total cells } \\
\left(\times 10^{\wedge} 6\right)\end{array}$ & $\begin{array}{l}\text { NK cells } \\
\left(\times 10^{\wedge} 6\right)\end{array}$ & $\begin{array}{l}\text { Total cells } \\
\text { (fold) }\end{array}$ & $\begin{array}{l}\text { NK cells } \\
\text { (fold) }\end{array}$ \\
\hline 1 & 67.5 & 5.3 & 3802 & 3681.9 & 56.3 & 694.7 \\
\hline 2 & 50.96 & 2.7 & 1102 & 871.7 & 21.6 & 322.9 \\
\hline 3 & 33.75 & 1.1 & 1651.9 & 1568.1 & 48.9 & 1425.5 \\
\hline $\begin{array}{c}\text { Average } \\
(\text { Mean } \pm \text { SD) }\end{array}$ & $50.7 \pm 18.9$ & $3.0 \pm 2.1$ & $2185.3 \pm 1426.8$ & $2040.6 \pm 1463.5$ & $42.3 \pm 18.3$ & $814.4 \pm 560.9$ \\
\hline
\end{tabular}

\section{Immunophenotyping Analysis by Flow Cytometry}

In average, the expanded NK cells (CD56+CD3-) presented $90.6 \% \pm 8.9 \%$ purify of immune cell population (ranging from $80.7 \%$ to $96.8 \%$ ). After 21 days of expansion culture, the percentage of NK cell increased by $17.8 \pm 9.3$ fold. There was a significant change in markers expression during culturing process (Figure 2A). The percentages of CD56dim CD3- cells at day 0 was $7.4 \pm 3 \%$. At the initial day, the CD56-CD3+ population was dominant, until day 3 the CD56+CD3-population started rising sharply and reached $>43 \%$ at day 5 . From day 3 to day 10 , the rate of NK cells increased dramatically and rapidly. From day 10 , the rate of NK cell continued to slowly increase and peaked at $88.5 \pm 11.5 \%$.
There were a gradually rise in the percentage of CD56bright CD3- NK cells. In average, the percentage of CD56bright CD3increased from $0.2 \% \pm 0.1 \%$ at the initial day and peaked at $84.2 \%$ $\pm 14.4 \%$ at the final culturing day. Simultaneously, we also observed that an increase in CD56 expression went parallel with a rise in the percentage of CD56 bright CD3- NK cells (Figure 2B). The more CD56 expression, a more number of CD56bright CD3- NK cells appeared in population. At the final day, the expression of CD56 was strongest led to the rate of CD56bright CD3- NK cells reached highest point at $94.4 \%$ (case 1 ) and $74 \%$ (case 2). In average, the percentage of CD56bright CD3- increased from $0.2 \% \pm 0.1 \%$ at the initial day and peaked at $84.2 \% \pm 14.4 \%$ at the final culturing day. 


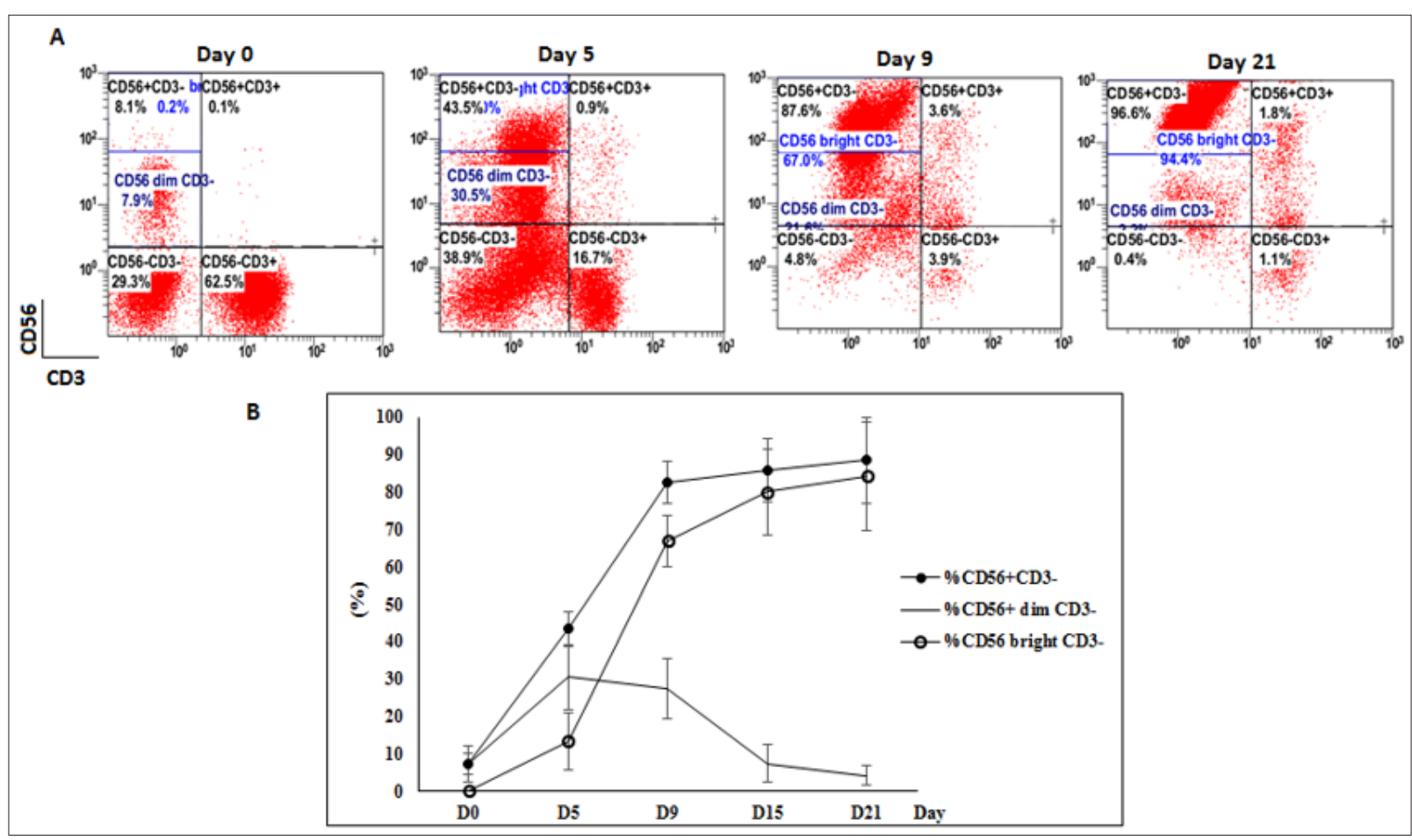

Figure 2: The phenotype of immune cell population. (A) The flow analysis of case 1 at day 0 , day 5, day 9 and day 21. (B) The correlation between the proportion of total NK cells CD56+CD3- and CD56 ${ }^{\text {dim }}$ CD3- and CD56 ${ }^{\text {bright }}$ CD3.

\section{Discussion}

The NK expansion kit BINKIT which we used for culturing includes initial flask, initial medium and initial cocktail. The initial flask is coated with anti-CD3 antibody and anti-CD16 antibody. These antibodies may bind to the surface receptors of cells other than NK cells (CD3, CD16) and then stimulating these cells to release many liquid growth factors which are needed for NK expansion. IL-2 is known as the most optimal for NK proliferation. Actually, IL-2 enhanced at least 10 -fold more NK cell expansion compared to IL4, IL-7, or IL-12 [11]. The initial cocktail contains IL-2 (Interleukin -2) which joins in signal pathways to proliferate NK cells and form the NK function. Concretely, these factors enhance the cytotoxicity of NK cells by stimulating the secretion of IFN- $\gamma$ which is needed for killing other cells (cancer cells or infected cells) [12]. The initial medium contains OK-432, this serve as an immune adjuvant capable of activating, for example, monocytes, through the binding to the surface TLR of the monocytes so that immune response is activated [13].

Another important component is zoledronic acid - a kind of bisphosphonate buffer. This acid inhibits the intracellular synthesis of Farnesyl Pyrophosphate (FPP), resulting in the accumulation of its precursor Isopentenyl Pyrophosphate (IPP). As a result, the immune response of the organism can reportedly be activated [13-15]. There are many different factors which are essential for stimulation culture as well as expansion culture process. In 2011, Jan Sphanholtz and his research group also reported their results about NK differentiation from CD34+ UCB cells. The CD34+ UCBderived cells were expanded for 2 weeks. From day 14, the cells were cultured in 2 ways. In the first way, they used the culture bag for CD34+ expansion and combine with medium containing SCF, IL-7, IL-15 and IL-2 within 6 weeks. As the result, the mean total cell expansion was 1300 fold, the NK cells product was 900$1900 \times 10^{\wedge} 6$ and \%CD56+CD3- was $71 \% \pm 9 \%$ [16]. Their process prolonged 6 weeks as opposed to our 3 week-process, which can lead to the cell exhausted as we observed at the end of the culture (3 weeks) the dominant of crooked cells. In the second way, they used a bioreactor system for cell culturing for 6 weeks and obtained much better results: the mean total cell expansion was 2100 fold, high pure NK product with $92 \% \pm 2 \%$ and total NK cells were 1600 $-3700 \times 10^{\wedge} 6$ [16].

As in Shah and his colleagues' report which was published in October 2013, the proportion of CD56+CD3- obtained was > 95\% and a 2389-mean fold expansion of NK cells derived from frozen UCB was achieved after 14 day. These results are much higher in comparison with our results. The main cause of this may be the fact that their method for UCB NK cells expansion was different from our approach. They use artificial antigen-presenting cell (aAPC) and cytokines including IL-2, IL-15 and FLT-3 ligand. The aAPC led to the generation of expanded UCB NK cells that displayed increased expression of NK cell activating receptors, increased perforin and granzyme expression [17]. These results are really impressive because although the time of culturing process is shorter, the number of NK cells and the rate of purify NK cells are both much higher than our results. This demonstrates that although there were some growth supplements which are the same as ours, the culturing conditions in Anushruti's procedure are more 
specific and effective for NK expansion. Similarly, Vasu S et al also expanded cord-blood NK cells using irradiated Epstein-Barr virustransformed lymphoblastoid feeder cell lines from only $1 \mathrm{ml}$ of umbilical cord unit. At day 21, the number of NK cells was $430 \times 106$ (range $44-4321 \times 106$ ) [18]. However, this method involved feeder layer, this led to the certain stage in which interaction between NK cells and strange cells. Therefore, it was difficult to control the influence of these feeder cells to NK cells. This is also a drawback of this method in comparison with our approach.

CD56bright have higher frequency in UCB as opposed to peripheral blood while CD56dim plays a reverse trend. In fact, upon stimulation with cytokines such as IL-2 or IL-12, not only the number but also the cytotoxic activity of CD56bright NK cell subsets dramatically increase. The percentage of NK cells in population was determined exactly by \%CD56+CD3-. Moreover, in 2004, Ferlazzo reported that culturing in IL-2 can induces not only the proliferation but also the efficient cytotoxic activity of CD56bright. Therefore, we focus on the change in \%CD56 bright to evaluate the increase of activated NK cells. Meanwhile, our culturing condition is the combination of IL-2 and some other growth factors such as antibodies... This contributed in the activation of NK cell. At initial day, the rate of CD56dim NK cells was dominant in population. From day 5 to the final day, the rate of CD56dim NK cells declined dramatically. Conversely, the proportion of CD56 bright rose significantly to $>90 \%$. This means that the function of NK cells had been modified comparable with initial stage. This also demonstrates that the growth supplements in our culturing condition were efficient in activating NK cells and orienting the function expression of CD56 bright NK cells.

\section{Conclusion}

Human cord blood has a significant potential to expand NK cells to relevant number for clinical use. We were success to expand NK cells from Vietnamese human cord-blood with significantly pure (more than 90\%), high viability (more than 98\%) and the number of NK cell were relevant to clinical application $\left(2040.6 \times 10^{\wedge} 6\right.$, ranging from 871.7 to $3681.9 \times 10^{\wedge} 6$ ). These NK cell products are suitable for clinical trials in order to apply in cancer treatment.

\section{Competing Interests}

The author(s) declare that they have no competing interests

\section{Acknowledgement}

This study was funded by Vingroup JSC (grant number ĐT-01)

\section{References}

1. Terunuma H, Deng X, Nishino N, Watanabe K (2013) NK cell-based autologous immune enhancement therapy (AIET) for cancer. Journal of stem cells \& regenerative medicine 9(1): 9-13.
2. Levy EM, Roberti MP, Mordoh J (2011) Natural killer cells in human cancer: from biological functions to clinical applications. BioMed Research International 2011: 676198.

3. Dahlberg CI, Sarhan D, Chrobok M, Duru AD, Alici E (2015) Natural killer cell-based therapies targeting cancer: possible strategies to gain and sustain anti-tumor activity. Frontiers in immunology 30(6): 605.

4. Abbas AK, Lichtman AH, Pillai S (2015) Cellular and Molecular Immunology. p. 65-69.

5. Cooper MA, Fehniger TA, Caligiuri MA (2001) The biology of human natural killer-cell subsets. Trends in immunology 22(11): 633-640.

6. Lanier LL, Le AM, Civin C, Loken M, Phillips J (1986) The relationship of CD16 (Leu-11) and Leu-19 (NKH-1) antigen expression on human peripheral blood NK cells and cytotoxic T lymphocytes. The Journal of Immunology 136(12): 4480-4486.

7. Jacobs R, Hintzen G, Kemper A, Beul K, Kempf S, et al. (2001) CD56bright cells differ in their KIR repertoire and cytotoxic features from CD56dim NK cells. European journal of immunology 31(10): 3121-3126.

8. Brunker PA (2012) Immunology IV: Clinical Applications in Health and Disease. Transfusion 52(8): 1836-1836.

9. Cheng M, Chen Y, Xiao W, Sun R, Tian Z (2013) NK cell-based immunotherapy for malignant diseases. Cellular \& molecular immunology 10(3): 230-252.

10. Sarvaria A, Jawdat D, Madrigal J, Saudemont A (2017) Umbilical Cord Blood Natural Killer Cells, Their Characteristics, and Potential Clinical Applications. Frontiers in immunology 8: 329.

11. Robertson MJ, Manley TJ, Donahue C, Levine H, Ritz J (1993) Costimulatory signals are required for optimal proliferation of human natural killer cells. The Journal of Immunology 150(5): 1705-1714.

12. Trinchieri G, Matsumoto Kobayashi M, Clark S, Seehra J, London L, et al. (1984) Response of resting human peripheral blood natural killer cells to interleukin 2. Journal of Experimental Medicine 160(4): 1147-1169.

13. Ryoma Y, Moriya Y, Okamoto M, Kanaya I, Saito M, et al. (2004) Biological effect of OK-432 (picibanil) and possible application to dendritic cell therapy. Anticancer research 24(5C): 3295-3302.

14. Gober HJ, Kistowska M, Angman L, Jenö P, Mori L, et al. (2003) Human T cell receptor $\gamma \delta$ cells recognize endogenous mevalonate metabolites in tumor cells. Journal of Experimental Medicine 197(2): 163-168.

15. Van Beek E, Pieterman E, Cohen L, Löwik C, Papapoulos S (1999) Farnesyl pyrophosphate synthase is the molecular target of nitrogencontaining bisphosphonates. Biochemical and biophysical research communications 264(1): 108-111.

16. Spanholtz J, Preijers F, Tordoir M, Trilsbeek C, Paardekooper J, et al. (2011) Clinical-grade generation of active NK cells from cord blood hematopoietic progenitor cells for immunotherapy using a closedsystem culture process. PloS one 6(6): e20740.

17. Shah N, Martin Antonio B, Yang H, Ku S, Lee D A, et al. (2013) Antigen presenting cell-mediated expansion of human umbilical cord blood yields log-scale expansion of natural killer cells with anti-myeloma activity. PloS one 8(10): e76781.

18. Vasu S, Berg M, Davidson Moncada J, Tian X, et al. (2015) A novel method to expand large numbers of CD56+ natural killer cells from a minute fraction of selectively accessed cryopreserved cord blood for immunotherapy after transplantation. Cytotherapy 17(11): 1582-1593. 
ISSN: 2574-1241

DOI: 10.26717/BJSTR.2018.08.001711

Hoang Thi My Nhung. Biomed J Sci \& Tech Res

(c) (9) This work is licensed under Creative Submission Link: https://biomedres.us/submit-manuscript.php

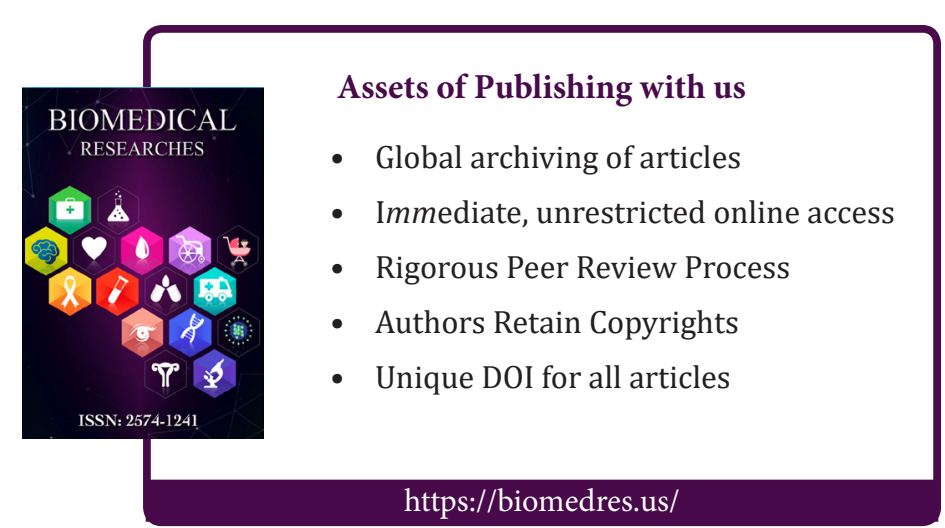

